\title{
Utilization of The Collector Two Colored Rainbow System in Istanbul
}

\author{
Z. $\mathrm{ER}^{*}$ \\ Istanbul Technical University, Faculty of Science and Letters (Maritime Faculty), \\ Physics Engineering Department, 34469 Maslak-Istanbul, Turkey
}

\begin{abstract}
Solar heating is known as one of the cleanest and cost-effective technologies that inherently reduces cost of energy and dependency on imported fuels while being applied and used in commercial and product applications in the industry. The modus operandi of this study introduces a procedure that was coined as "Two Colored Rainbow" which is used to represent two different applications using by solar tracer, rather than the direct application of solar thermal system. Two rainbow system traps the heat from the sun (solar radiation) and transfer the heat to water or to air for use as thermal energy by the aid of solar panels that the system is coupled with solar tracer. The vacuum tube and flat type collectors were used as an experimental portion of this study while the effective utilization of solar energy on a daily basis has been identified by the algorithm that has been developed in $\mathrm{C} \#$ to determine how many percentage of an hotel's energy in Istanbul which serves up to five hundred guest capacity need could be compensated by providing optimum size and quantity of solar collectors.
\end{abstract}

DOI: $10.12693 /$ APhysPolA.128.B-300

PACS: 96.60-j, 96.60.Ub, 96.60.Q-

\section{Introduction}

Conventional solar water heating system is composed of solar collectors and a water tank [1]. The solar collectors absorb the heat by solar radiation to heat up water, either directly or indirectly. Obtained hot water is stored in the storage tank for subsequent use [2-5]. An alternative arrangement is to use a packaged type solar water heater, with solar collectors and an insulated storage tank packaged together in one integrated unit. Therefore the solar collectors fall into two general categories based on its design, i.e. flat-plate collectors and evacuated tube collectors.

Two rainbow system traps the heat from the sun (solar radiation) and transfer the heat to water for use as thermal energy, while collector panels are being fitted with solar tracer system. The system which was used in this study and was demonstrated with this study goal diagram in Fig. 1 that overarches the solar water heating and solar space heating by the aid of solar panels.

\section{Calculations of solar angles using $\mathrm{C} \#$ code}

The vacuum tube and flat type collectors were used in experimental part of this study. The effectiveness utilization of solar energy in terms of each day has been identified and computed by the algorithm that has been developed in $\mathrm{C} \#$ to determine the how many percentage of the energy for an hotel in Istanbul that serves to five hundred guest capacity need could be compensated with providing optimum size and quantity of solar collectors. Calculations for the determination of solar evaluations such as azimuth angle, sun set etc. have been computed

\footnotetext{
*e-mail: erzuh@itu.edu.tr
}

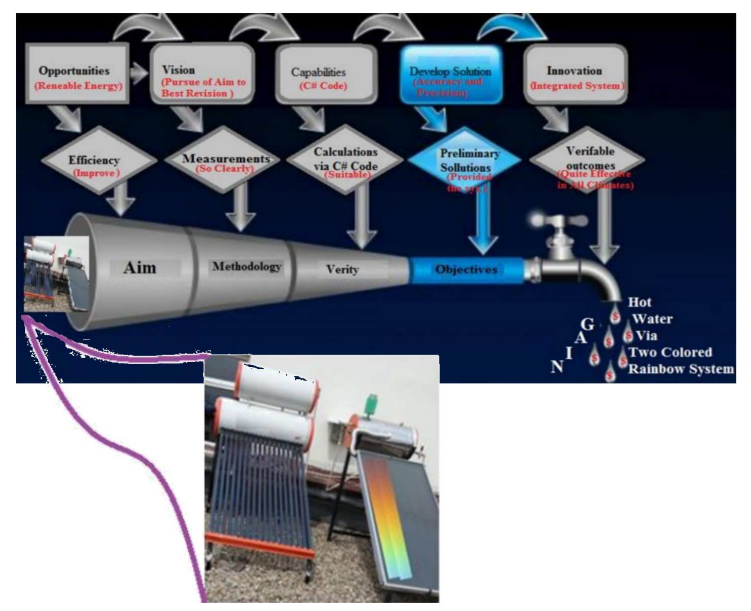

Fig. 1. The research goals and the two colored rainbow system at Faculty of Science and Letters, ITU in Istanbul.

to be able to recognize utilization of solar power for the energy demand of the aforesaid mentioned hotel.

In these premises useful relationships for the angle of incidence of surfaces sloped due north or due south can be derived from the fact that surfaces with slope $\beta$ to the north or south have the same angular relationship to beam radiation as a horizontal surface at an artificial latitude of $\varphi-\beta[4,6]$. Subsequently the incidence angle $(\theta)$ Eq. (1) yields to the computation $[5,7,8]$

$\cos \theta=\cos (\varphi-\beta) \cos \delta \cos \omega+\sin (\varphi-\beta) \sin \delta$,

where $\varphi$ is terrestrial latitude, $\delta$ - current solar declination, $\theta$ - incidence angle, $\beta$ - sloped angle, $\omega$ - hour angle.

The zenith angle of the sun $\left(z_{H}\right)$ and the altitude of the sun $\left(A_{H}\right)$ above the horizon were computed by Eqs. (2) and (3) respectively $[5,7,8]$ 


$$
\begin{aligned}
& z_{H}=\arccos (\sin \varphi \sin \delta+\cos \varphi \cos \delta \cos \tau), \\
& A_{H}=\arccos \left(\frac{\cos \varphi \sin \delta-\sin \varphi \cos \delta \cos \tau}{\sin z_{H}}\right),
\end{aligned}
$$

where $z_{H}$ is the zenith angle of the sun, $A_{H}$ - the altitude of the sun above the horizon. Additionally

$$
\tau=\frac{n}{6} \arccos (-\tan \varphi \tan \delta),
$$

where $n$ is the difference between official and local time.

On the southern hemisphere the modification of Eq. 1 is necessary. $\varphi-\beta$ is to be replaced by $\varphi+\beta$, consistent with the sign conventions on $\varphi$ and $\delta$. Then the equation becomes as follows $[5,7,8]$

$$
\cos \theta=\cos (\varphi+\beta) \cos \delta \cos \omega+\sin (\varphi+\beta) \sin \delta
$$

and the Eq. (1) can be solved for the sunset hour angle $\omega_{s}$, when $\theta_{z}=90^{\circ}$

$$
\cos \omega_{s}=-\frac{\sin \varphi \sin \delta}{\cos \varphi \cos \delta}=-\tan \varphi \tan \delta .
$$

The sunrise hour angle is opposite to the sunset hour angle. It also follows that the daylight time is [9-12]

$$
N=\frac{2}{15} \arccos (-\tan \varphi \tan \delta) \text {. }
$$

An additional angle of interest is the profile angle of beam radiation on a receiver plane $R$ that has a surface azimuth angle of $\gamma$. It is the projection of the solar altitude angle on a vertical plane perpendicular to the plane $[8,11,12]$. The profile angle is useful in calculating shading by overhangs and can be determined from Eq. (10) [8-10]:

$$
\tan \alpha_{p}=\frac{\tan \alpha_{s}}{\cos \left(\gamma_{s}-\gamma\right)}
$$

Solar azimuth angle of $\gamma_{s}$ is calculated using by:

$$
\tan \gamma_{s}=\frac{\sin \omega}{\sin \varphi \sin \omega-\cos \varphi \tan \delta} .
$$

The solar angles which are calculated by the computer code were compared with the solar intensities which are measured by pyranometer. The brief illustration of obtained figures was pointed out below.

The period of experimental compared to the computation was selected in between January 01, 2014, and July 31, 2014, to determine the amount of energy during winter and spring seasons. Therefore the sample computational data together with its experimental output were illustrated below for the sample two days such as February 13th, April 2nd and July 1st to precisely describe the method of evaluation between experimental data and the computed theoretical output representing the most coldest and hottest day in the biannual period. The zenith and solar azimuth angles were calculated as $\varphi=41^{\circ} 1^{\prime} 2^{\prime \prime}$ at a 9:30 am on February 13th and 6:30 pm on July 1st, where $\delta=-13.87^{\circ}$ and $\omega=-40.73^{\circ}$. Similarly the calculation of the time of sunrise, solar altitude, zenith, solar azimuth, and profile angles for a $60^{\circ}$ sloped surface facing $25^{\circ}$ west of south at 06:08 pm solar time on March 16 th at altitude of $41^{\circ} 1^{\prime} 2^{\prime \prime}$ is determined using Eq. (8) as $\delta=-2.33^{\circ}$ and $\omega_{s}=87.97^{\circ}$.
Furthermore other calculation examples such as on April 2nd and July 1st at altitude of $41^{\circ} 1^{\prime} 2^{\prime \prime}$ were collected in the database. Those data consist the calculation results such as declination angles at $4.50^{\circ}$ and $2311^{\circ}$; sunsets at $93.93^{\circ}$ and $111.79^{\circ}$; local sunrise time of $06: 52$ and 05:39; local sunset time of 19:23 and 20:34; sun clock sunrise time of 05:44 and 04:32; sun clock sunset time of 18:15 and 19:27 and also day length of 12 hour 31 minutes and 14 hour 55 minutes with respectively for April 2nd and July 1 st at altitude of $41^{\circ} 1$ '2'. The daily based and monthly average figures have been obtained throughout the biannual framework with the similar congruity and the data that were obtained experimentally has been compared for the determination of its consistency.

\section{Results and conclusion}

This study was aimed to offer the optimization of the design requirements and the recognition of probable interferences during the operation of the system. The calculation of data is not restricted to daily figures but can be performed for specified time intervals throughout a day.

The guidelines obtained in this study for the solar system design process could be expressed as follows. The temperature increase in network supply water, the solar intensity and the duration of exposure to sunlight rays per day time are highest during summer season and the demand of hot water is highest in winter season. It must be taken into account by estimation of the energy delivery by the system. The design pattern has to prevent the system being idle during summer season by choosing the ideal number of collectors together with the optimum storage volume balance. All in all the system design pattern should be contemplated in such a manner that the daily energy consumption rates should be consistent with the annual/biannual simulation data.

Consequently the energy demand of five hundred guest hotel in Istanbul was determined for each month in biannual period and the simulated data have yielded with the $69 \%$ of energy demand could be covered by solar energy in April while outfitting 171 collectors and the hundred percent of the energy demand could be compensated via by the arrangement of 241 collectors oriented as follows: declination angle $4.50^{\circ}$, elevation angle $53.48^{\circ}$ and the azimuth angle $0.00^{\circ}$ at $12: 00$ hours in sun hour (with the local time 13:08). The experimental results yielded that the solar intensity for the aforesaid month was found $809 \mathrm{~W} / \mathrm{m}^{2}$ during the maximum intensity of sun at 12:40 local time. In these premises the simulated data and the experimental results demonstrated that the simulated data at 13:08 hours have not pointing out the actual azimuth angle which the foreknown reality would be used to compare the experimental data especially for the considerably low insolation intensity in winter and other seasons. Therefore the complementary information from experimental data should be reiterated into the simulation to enhance results. 


\section{References}

[1] Residential Compliance Manual, Water Heating Requirements, California Energy Commission, Sacramento 2013.

[2] Guide to Meteorological Instruments and Methods of Observation, WMO-No. 8, WMO, Geneva 2008.

[3] K. Crumbaker, P. McCarty, Introduction to Domestic Solar Hot Water Systems, Fact Sheet No. 10.627, Consumer Series Energy, Colorado State University, Fort Collins 2013.

[4] A. Christopher, P.E. Homola, Solar Domestic Hot Water Heating Systems Design, Installation and Maintenance.

[5] A. Szymanowska, M.Sc. Thesis, University of Iceland \& University of Akureyri, Akureyri 2011.

[6] E.J. Urban, M.Sc. Thesis, Appalachian State University, Boone 2011.

[7] C.J. Chen, Physics of Solar Energy, Wiley, New Jersey 2011.
[8] J.A. Duffie, W.A. Beckman, Solar Engineering of Thermal Processes, Wiley, Wisconsin 1980.

[9] A. Waggott, N. Pearsall, K. Theobald, S. Walker, Monitoring Useful Solar Fraction in Retrofitted Social Housing.

[10] Measurement of Sunshine Duration Prelim2014Ed_P-I_Ch-8, WMO, Geneva 2014.

[11] G. Major, Radiation and Sunshine Duration Measurements, Comparison of Pyranometers and Electronic Sunshine Duration Recorders of RA VI Budapest, July-December 1984, WMO\&TD-No. 146, WMO, Geneva 1986.

[12] E. Vuerich, J.P. Morel, S. Mevel, J. Oliviéri, Updating And Development of Methods for Worldwide Accurate Measurements of Sunshine Duration, Teco-2012, Brussels 2012. 